\title{
Atomic hydrogen concentration profiles at filaments used for chemical vapor deposition of diamond
}

\author{
L. Schäfer and C.-P. Klages \\ Philips GmbH Forschungslaboratorium Hamburg, Vogt-Kölln-Strasse 30, W-2000 Hamburg 54, Germany \\ U. Meier and K. Kohse-Höinghaus \\ DLR-Institut für Physikalische Chemie der Verbrennung, Pfaffenwaldring 38-40, W-7000 Stuttgart 80, \\ Germany
}

(Received 13 July 1990; accepted for publication 8 November 1990)

\begin{abstract}
The quantitative determination of atomic hydrogen concentrations $c_{\mathrm{H}}$ in the vicinity of hot filaments is performed with two-photon laser-induced fluorescence. The measurements yield atomic hydrogen concentration profiles up to $28 \mathrm{~mm}$ from the filament surface with a spatial resolution of about $0.5 \mathrm{~mm}$. The nonequilibrium nature of the hydrogen dissociation on the filament surface results in a saturation of hydrogen concentration profiles $c_{\mathrm{H}}(r)$ for gas pressures above 10 mbar. Atomic concentrations in immediate vicinity of the filament are significantly lower than expected from thermodynamical calculations and depend on the filament diameter. Addition of methane results in a decrease of $c_{\mathrm{H}}$ by more than $30 \%$ near the filament and a steeper $c_{H}(r)$ dependence, demonstrating the accelerated consumption of $\mathrm{H}$ atoms by the presence of hydrocarbon species. $\mathrm{H}$ concentration profiles for $\mathrm{Ta}, \mathrm{Ir}$, and $\mathrm{W}$ filaments show a dependence on filament materials which might be taken into account when selecting filament materials for chemical vapor deposition of diamond.
\end{abstract}

For the formation of synthetic diamond several chemical vapor deposition (CVD) methods, using thermal or plasma activation of the gas phase, are now established. ${ }^{1-6}$ A common feature of all methods is an abundance of molecular hydrogen in the gas phase. Although the detailed mechanisms leading to diamond formation are not yet understood, the importance of the presence of hydrogen for selecting diamond formation is widely accepted. Several possible roles within the mechanism of diamond formation have been attributed to hydrogen. Hydrogen molecules are supposed to prevent the formation of polycyclic aromatic hydrocarbons in the gas phase which otherwise lead to the deposition of nondiamond carbon phases. ${ }^{7}$ On the other hand there are several ways for atomic hydrogen, created by the activation of the gas atmosphere, to influence the deposition process. $\mathrm{H}$ atoms may react with hydrocarbons, which together with molecular hydrogen usually constitute the source gas, to form hydrocarbon species like methyl or acetylene in the gas phase. These species are by some authors assumed to be key species in the process of diamond formation. ${ }^{8,9} \mathrm{By}$ its ability to etch nondiamond carbon phases at the growth front much faster than diamond ${ }^{10}$ and to create new growth sites, atomic hydrogen may lead to the formation of thermodynamically metastable diamond as the only phase. ${ }^{11}$ Furthermore, $\mathrm{H}$ atoms bonded to carbon on the surface may stabilize the $s p^{3}$ bonds necessary for diamond formation. ${ }^{12}$

The dependence of atomic hydrogen concentrations on methane addition and filament temperature in a filamentassisted diamond deposition system has been investigated using resonance-enhanced multiphoton ionization (REMPI). ${ }^{12}$ Although this technique offers a good spatial resolution, a quantitative determination of $\mathrm{H}$ concentrations was not possible because of the lack of a calibration for the REMPI signals.
We determined absolute $\mathrm{H}$ concentration profiles at hot filaments under typical diamond CVD conditions by a two-photon laser-induced fluorescence (LIF) method used earlier for the detection of $\mathrm{H}$ atoms in low-pressure flames. ${ }^{13}$ The applicability of LIF for different pressures, methane fractions, and varying filament temperature, diameter and material used in filament-assisted CVD of diamond is demonstrated by Meier et al. ${ }^{14}$ Reference 14 also contains a detailed description of the experimental setup and the calibration of the LIF signals.

Radial temperature profiles, necessary for the determination of calibration factors accounting for quenching and Doppler broadening of the LIF signal, were estimated using a model introduced by Langmuir and coworkers for the description of heat losses from hot filaments to the surrounding gas phase. ${ }^{15,16}$ The temperature estimation includes a temperature drop at the filament over the first mean free path of hydrogen molecules. This temperature drop depends on gas pressure and filament radius. For example at $2620 \mathrm{~K}$ filament temperature, $1 \mathrm{~mm}$ filament radius, and a pressure of $30 \mathrm{mbar}$, the resulting radial gas temperature profile varied from about $2500 \mathrm{~K}$ ncar the filament to about $1050 \mathrm{~K}$ at the border of the detection region $28 \mathrm{~mm}$ from the filament. A temperature profile measured by Harris and Weiner ${ }^{17}$ with a thermocouple also yields a temperature drop near the filament of about $500 \mathrm{~K}$. Although their experimental conditions are not exactly the same-especially the radius of their filament is not mentioned-the decrease in the gas phase seems to be smoother than our estimated ones. On the other hand, the calibration factors for quenching and Doppler broadening nearly compensate each other for temperatures above 1200 $\mathrm{K} .{ }^{14}$ Therefore it is obvious that the cstimation of the tcmperature profiles is not critical for the $\mathrm{H}$ concentration profiles determined by the LIF method at the experimental 


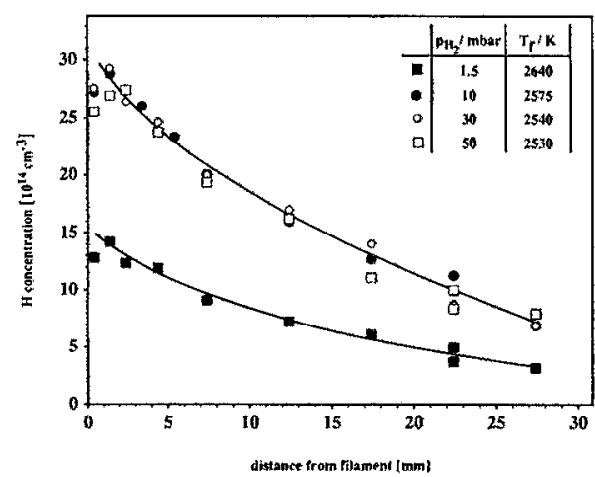

FIG. 1. Atomic hydrogen concentrations vs distance from the Ta filament surface for different molecular hydrogen pressures. The filament diameter is $2.0 \mathrm{~mm}$ in each case.

conditions employed here.

With regard to statistical and systematical errors in the calibration the uncertainty in the determination of absolute values for atomic hydrogen concentrations is about a factor of 2, whereas the error for relative concentrations measured at equal pressures, that means equal quenching conditions, is approximately $10 \%$, mainly given by the statistical error due to signal fluctuations.

Atomic hydrogen concentrations for different pressures are presented in Fig. 1 for positions up to a distance of $28 \mathrm{~mm}$ from the tantalum filament surface. As the heating power was kept constant in these experiments, the filament temperatures, measured with a two-wavelength $(0.87$ and $0.90 \mu \mathrm{m})$ pyrometer, decrease with increasing pressure due to growing conductive heat losses to the gas phase. Figure 1 shows a saturation of atomic hydrogen concentrations at pressures in excess of $10 \mathrm{mbar}$ (several data points measured at 100 mbar, not shown in Fig. 1, yield the same $\mathrm{H}$ concentration), at variance with the trends of chemical equilibrium concentrations $c_{\mathrm{H} \text { eq }}$, calculated using thermodynamic data from literature, ${ }^{18}$ which show a steady increase of $c_{\mathrm{H}, \mathrm{eq}}$ roughly proportional to the square root of pressure. At $1.5 \mathrm{mbar}$ the measured concentrations near the filament are about $50 \%$ of the equilibrium values calculated for filament temperature. The deviations grow larger with increasing pressure until, at $100 \mathrm{mbar}$, experimental values are only $12 \%$ of the equilibrium data.

The increase of $c_{\mathrm{H}}$ with temperature (see Fig. 2) is nearly equal to what is suggested by equilibrium considerations, but absolute values are only about $25 \%$ of $c_{\mathrm{H}, \mathrm{eq}}$, again calculated for filament temperatures. However the temperature dependence of $c_{\mathrm{H}}$ appears to be stronger than suggested by the relative $\mathrm{H}$ concentration measurements of Celii and Butler. ${ }^{12}$

With decreasing filament area per unit filament length, the hydrogen atom generation rate per unit area of the filament surface must increase in the case of thermal equilibrium in order to keep pace with the diffusion flux away from the filament, which is only slowly varying with the filament diameter. ${ }^{19}$ Kinetic limitations of the dissociation reaction will otherwise lead to decreasing surface concentrations with decreasing flament radius. Experimentally,

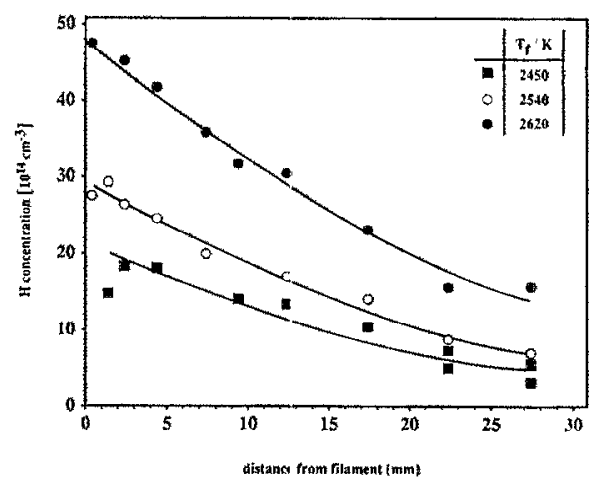

FIG. 2. Atomic hydrogen concentration profiles for a $2.0-\mathrm{mm}$-diam Ta filament at different temperatures $T_{f}$. The molecular hydrogen pressure is 30 mbar in each case.

this effect becomes evident from a comparison of the concentration profiles lor 0.3 - and 2.0-mm-diam filaments, respectively, at $2700 \mathrm{~K}$ (see Fig. 3). Because of their different filament temperatures the deviation between the profiles for 1.0 and $2.0 \mathrm{~mm}$ diameter, on the other hand, can be attributed to the temperature difference alone.

An increasing power input per unit area is generally necessary in order to compensate for the heat losses due to conduction or the generation of energetic species on the filament surface, when its diameter grows smaller. That is demonstrated by the experimental results of Jansen $e t$ al. ${ }^{20}$ Unlike these authors, we could not verify smaller radius filaments to become "increasingly more energy efficient," a conclusion which had been drawn from a numerically treated theoretical model of hydrogen dissociation and diffusion in the vicinity of a hot filament. An analytical treatment of this model is possible, if the dissociation is confined to the filament surface (exclusion of gas phase dissociation, limiting case with $\alpha=0$ in terms of the cited paper). The quantity "Atomic Hydrogen per Unit Input Power" (Figure 7 of Ref. 22) can then be shown to be proportional to $R^{2} / D, R$ and $D$ being the reactor radius and hydrogen diffusion coefficient, respectively, but it is independent of the filament radius $r_{0}$, if quantities of second order in $r_{0} / R$ are neglected. In practice this approxi-

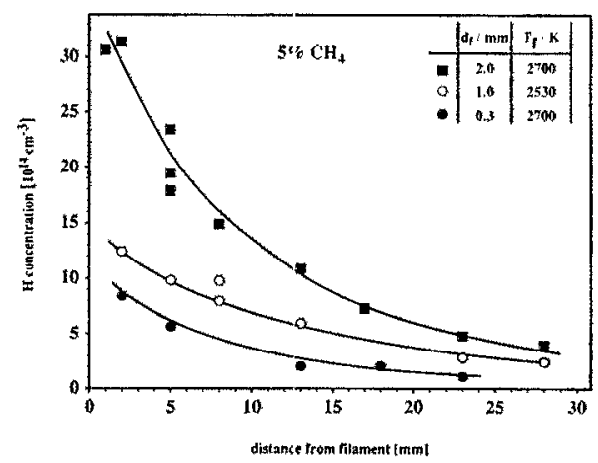

FIG. 3. Profiles of atomic hydrogen concentrations for Ta filaments with different diameter $d_{f}$. The gas phase contains $5 \%$ methane at a total pressure of 30 mbar. 
mation is generally justified.

The influence of methane addition on $\mathrm{H}$ concentration profiles was studied in order to test a hypothesis according to which in HFCVD of diamond the detrimental effect of too large methane concentrations (typically $0.5 \%$ ) on the phase purity of the growing film is due to an acceleration of the total hydrogen consumption in the gas phase by addition of methane. Hydrogen abstraction processes can open new channels for reactions of atomic hydrogen in addition to the relatively slow three-body recombination. The calculations performed by Frenklach indicate an accelerated consumption of $\mathrm{H}$ atoms in a $3 \%$ methane/hydrogen mixture quenched from 2350 to about $600{ }^{\circ} \mathrm{C}$ within $1 \mathrm{~s}$, compared with a mixture containing only $0.3 \%$ methane (see Fig. 2 of Ref. 7).

The experiments were conducted with Ta filaments which are superficially converted to the gold-colored carbide $\mathrm{TaC}$ upon contact with hydrocarbons. At 5 vol \% methane the resulting change of optical emissivity leads to an $80 \mathrm{~K}$ increase of $T_{f}$ at constant electrical power (cp. Figs. 2 and 3 ). Nevertheless the maximum $\mathbf{H}$ concentration near the filament is more than $30 \%$ lower after the addition of methane. Simultaneously, the concentration profile becomes steeper, indicating the expected accelerated consumption of $\mathrm{H}$ in the gas phase. Based on the present results, one cannot exclude additional contributions of surface effects, which Celii and Butler ${ }^{12}$ put forward in order to explain their result of a decreasing REMPI signal upon methane additions.

The finding that kinetic factors determine the surface concentrations of atomic hydrogen on hot filaments raises the question for the efficiency of hydrogen dissociation in filaments made from different materials. Profiles for Ta, Ir, and $W$ filaments indicate that the Ta filament (carburized) generates somewhat higher $\mathrm{H}$ concentrations than the Ir filament, heated nearly to its melting point. The tungsten filament, however, yields the smallest deviation from the equilibrium value. For its practical use in hot-filament diamond CVD processes, however, the selection of a filament material is not governed by the hydrogen dissociation efficiency alone. Other critical factors are the mechanical and chemical stability under process conditions as well as contamination of the growing film by evaporating filament material.

Although the measured $\mathrm{H}$ atom concentrations are generally smaller than equilibrium values calculated for filament temperatures, they are in fact-except for a small region of a few $\mathrm{mm}$ diameter around the filamentssuperequilibrium concentrations, if comparison is made with the estimated gas temperatures. A numerically evaluated kinetic model including diffusion, thermodiffusion, dissociation, and recombination within the Langmuir-type film shows that the concentration profiles of $\mathbf{H}$ atoms are predominantly governed by their rapid diffusion to the film boundary, the recombination reaction being much too slow to restore chemical equilibrium in the gas phase. ${ }^{21}$

In conclusion, the quantitative determination of atomic hydrogen concentrations using two-photon laserinduced fluorescence yields kinetic limitations for hydrogen dissociation under conditions of HFCVD of diamond. The deviation from thermal equilibrium is confirmed by subequilibrium values of $c_{\mathrm{H}}$ near the filament and the dependence of $c_{\mathrm{H}}$ on filament geometry and material. One has to note that the presence of a substrate will change the concentration profiles. The presented results are, however, in good agreement with values published by Harris and Weiner. ${ }^{22}$ The effect of methane addition on the measured $\mathrm{H}$ profiles can be attributed to the consumption of atomic hydrogen by the hydrocarbon species in the gas phase, possibly in addition to surface effects due to the reaction of methane with the filament.

The authors would like to thank U. Bringmann for technical assistance. The work was supported in part by the Bundesministerium für Forschung und Technologie, grant No. 13N5607.

${ }^{1}$ R. C. DeVries, Ann. Rev. Mater. Sci. 17, 161 (1987).

${ }^{2}$ K. E. Spear, J. Am. Ceram. Soc. 72, 171 (1989).

${ }^{3}$ M. Kamo, Y. Sato, S. Matsumoto, and N. Setaka, J. Cryst. Growth 62, 642 (1983).

${ }^{4}$ S. Matsumoto, Y. Sato, M. Kamo, and N. Setaka, Jpn. J. Appl. Phys. 21, L183 (1982).

${ }^{5}$ K. Kurihara, K. Sasaki, M. Kawarada, and N. Koshino, Appl. Phys. Lett. 52, 437 (1988).

${ }^{6}$ L. M. Hanssen, W. A. Carrington, and J. E. Snail, Mater. Lett. 7, 289 (1989).

${ }^{7}$ M. Frenklach, J. Appl. Phys. 65, 5142 (1989).

${ }^{8}$ M. Tsuda, M. Nakajiama, and S. Oikawa, J. Am. Chem. Soc. 108, 5780 (1986).

${ }^{9}$ D. Huang, M. Frenklach, and M. Maroncelli, J. Phys. Chem. 92, 6379 (1988).

${ }^{10}$ N. Setaka, J. Mater. Res. 4, 664 (1989).

${ }^{1}$ B. V. Spitsyn, L. L. Bouilov, and B. V. Derjaguin, J. Cryst. Growth 52, 219 (1981).

${ }^{12}$ F. G. Celii and J. E. Butler, Appl. Phys. Lett. 54, 1031 (1989).

${ }^{13}$ U. Meier, K. Kohse-Höinghaus, and Th. Just, Chem. Phys. Lett. 126, 567 (1986).

${ }^{14}$ U. Meier, K. Kohse-Höinghaus, L. Schäfer, and C.-P. Klages, Appl. Opt. (to be published).

${ }^{15}$ K. B. Blodgett and I. Langmuir, Phys. Rev. 40, 78 (1932), and references therein.

${ }^{16}$ E. J. Covington, Illuminating Eng. 63, 134 (1968).

${ }^{17}$ S. J. Harris and A. M. Weiner, Appl. Phys. Lett. 53, 1605 (1988).

${ }^{18}$ R. Duff and S. H. Bauer, J. Chem. Phys. 36, 1754 (1962).

${ }^{19} \mathrm{~J}$. Crank, The Mathematics of Diffusion (Oxford University, London, 1956).

${ }^{20}$ F. Jansen, I. Chen, and M. A. Machonkin, J. Appl. Phys. 66, 5749 (1989).

${ }^{21}$ L. Schäfer, U. Bringmann, C.-P. Klages, U. Meier, and K. KohseHöinghaus, to be published in Diamond and Diamond-Like Films and Coatings, edited by J. C. Angus, R. E. Clausing, L. L. Horton, P. Koidl (Plenum, New York).

${ }^{22}$ S. J. Harris and A. M. Weiner, Appl. Phys. Lett. 55, 2179 (1989). 